\title{
Patientenaufklärung bei Jugendlichen
}

Darf man Minderjährige ohne Einwilligung der Eltern behandeln? Wen muss man aufklären - die Eltern oder das Kind? Und: Wie steht es um die ärztliche Schweigepflicht bei Minderjährigen? Die gesetzlichen Regelungen sind nicht immer eindeutig. Doch es gibt Eckpunkte, an denen sich der behandelnde

Arzt orientieren kann.

Lesen Sie am Beispiel eines konkreten Falles,

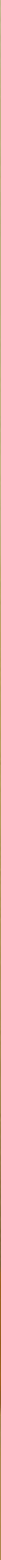
worauf Sie achten sollten. 
A Is Barbara Windfuhr*an jenem Nachmittag den Behandlungsraum betritt, erwartet sie ein nicht ganz alltäglicher Fall: Ronny, ein 15-jähriger Teenager, sein linker Arm ist rot-violett und dick geschwollen. „Ein Schlangenbiss“, kommt der Junge den Fragen der Ärztin zuvor. Die Kollegen der chirurgischen Notfall-Aufnahme haben ihn bereits erstversorgt, dann aber $\mathrm{zu}$ ihr in die Innere Abteilung geschickt.

\section{Falldarstellung}

Schlangenbiss Aus der Aufnahme bringt Ronny folgende Informationen mit:

- Zustand nach Schlangenbiss am linken Unterarm

- Zustand nach venöser Staubinde über $3 \mathrm{~h}$

- medikamentöse Schmerzbehandlung

- zunächst keine weitere Lokaltherapie indiziert

- Gefahr eines Kompartment-Syndroms, daher den Arm stündlich kontrollieren

- Bitte mögliche systemische Giftwirkungen abklären!

Halb trotzig, halb verunsichert berichtet Ronny über den Unfall: Er lasse seine Giftschlange nachmittags immer frei in seinem Zimmer kriechen. Diesmal habe sie ihn beim Einfangen in den Arm gebissen, woraufhin er den Arm sofort abgebunden habe - das müsse man schließlich machen nach Schlangenbissen, er kenne sich da aus.

Ins Krankenhaus sei er erst gegangen, als sich sein ganzer Arm verfärbt habe, stark angeschwollen sei und die Schmerzen nicht nachließen. Auch sei ihm etwas übel und schwindelig.

Giftnotruf Ein Schlangenbiss verlangt rasches Handeln. Um die Gefahr besser einschätzen zu können, ruft die Internistin einen Giftnotruf an. Gut, dass Ronny sein Haustier beim Namen kennt: Aspisviper. So kann die Giftzentrale sehr gezielt informieren:

- Die Aspisviper gibt zur Abwehr oft nur wenig Gift ab.

- Meist beschränken sich die Symptome auf Bissmarken und lokale Ödeme.

- Durch die Staubinde verblieb das Gift jedoch deutlich länger im Arm und

- führte vermutlich daher zu der starken Lokalwirkung.

Die gastrointestinalen Beschwerden und der Schwindel gehören bereits zu einer mittelschweren Vergiftung mit systemischer Wirkung. Selten gibt es auch aller- gische Reaktionen bis hin zum Schock, die in einzelnen Fällen protrahiert auftreten können [1].

Praxistipp Nummern für den Giftnotruf sind zum Beispiel:

- Berlin: 030/19240

- Bonn: 0228/19240

- Freiburg: 0761/19240

- Göttingen: 0551/19240

- München: 089/19240

Die Internet-Seiten der Informationszentralen finden Sie unter www.klinitox.de

Therapie Die Giftzentrale empfiehlt vor allem eine symptomatische Behandlung durch

- Hochlagern und Ruhigstellen des Armes sowie

- Fortführen der Schmerzbehandlung. Sollte die Schwellung jedoch auf den Rumpf übergehen oder sich der Allgemeinzustand verschlechtern, so sei

- unbedingt ein modernes Antiserum zu bestellen,

- dieses so schnell als möglich liefern zu lassen und

- bei gegebener Klinik in jedem Falle zu verabreichen.

Noch ist Ronnys Zustand stabil, und die Schwellung ist auf den Arm begrenzt. Um aber für einen progressiven Verlauf gewappnet zu sein, wendet sich die Ärztin in Absprache mit dem Oberarzt an das nächstgelegene Depot für Antiseren - es liegt gute $200 \mathrm{~km}$ entfernt. Für einen schnellen Transport organisiert Dr. Windfuhr die Feuerwehr und lässt das Antiserum mit Blaulicht bringen.

Ankunft der Mutter Die chirurgische Notaufnahme hatte Ronnys Eltern bereits über die Verletzung ihres Sohnes informiert - nun erscheint die Mutter im Krankenhaus. Sie wirkt zugleich besorgt und verärgert. Ronny scheint wenig begeistert über ihr Erscheinen.

Erst jetzt wird Barbara Windfuhr bewusst, dass der 15-Jährige ganz allein in die Klinik gekommen ist. In der Eile hatte sie Ronny gar nicht nach seinen Eltern gefragt - zumal er ihr durchaus verständig genug erschien, um das ärztliche Prozedere mit ihm allein zu besprechen.

Illegale Schlangenhaltung Die Wartezeit auf das Antiserum nutzt die Ärztin, um ihren Patienten auf eine weitere interessante Information der Giftzentrale anzusprechen:

- Die Aspisviper gehört zu den besonders geschützten Tierarten in Deutschland. Sie darf nur in Einzelfällen gehalten werden [2].

Ronny wendet sich zur Wand, seine Mutter nestelt an ihrer Handtasche herum. Im Flüsterton lässt Ronny vernehmen, dass bloß niemand auf die Idee kommen solle, ihm die Schlange wegzunehmen. Er will sich plötzlich nichts mehr sagen lassen und drängt nach Hause: „Es geht schon wieder, ich geh jetzt heim!“ Für Ronny steht augenscheinlich fest: Die Schlange, nicht er, bedarf jetzt großer Sorge.

Widerstand Dr. Windfuhr versucht, sich auf den jugendlichen Patienten einzulassen. Ruhig aber deutlich macht sie ihm die Sachlage noch einmal klar:

- Ronny wurde vor wenigen Stunden von einer Giftschlange gebissen.

- Der ganze Arm ist stark ödematös und bedarf dringend der Überwachung.

- Die Schmerzen sind nur durch das Analgetikum reduziert.

- Die systemischen Giftwirkungen nehmen möglicherweise noch zu.

Auch eine verspätete anaphylaktische Reaktion ist nicht auszuschließen [1]. Die Ärztin empfiehlt daher dringend eine Überwachung über mindestens weitere 6 h. Doch Ronny gibt sich unbeeindruckt: „Hey, ich bin 15 ! Ich weiß was ich tue und kann das selbst entscheiden!“

Selbstentlassung Ronnys Mutter ist klar auf ärztlicher Seite. Sie versucht ebenfalls, ihren Sohn zum Bleiben zu überreden - ohne Erfolg: Er packt bereits seine Sachen. Barbara Windfuhr entscheidet sich, den Patienten gehen zu lassen. Sie klärt Ronny und seine Mutter noch einmal über die möglichen Gefahren und Konsequenzen seines Handelns auf. Ihr dringender Rat:

- Falls sich der Gesundheitszustand in den nächsten Stunden verschlechtert, soll Ronny unbedingt sofort das Krankenhaus aufsuchen.

Sowohl Ronny als auch seine Mutter unterschreiben eine Erklärung, dass er gegen ausdrücklichen ärztlichen Rat das Krankenhaus verlässt - dann gehen sie. Eine Stunde später ist das Antiserum da.

\section{Juristischer Hintergrund}

Kind? Jugendlich? Minderjährig? Im vorliegenden Fall ist der Patient noch nicht erwachsen - doch wie nennt man einen Nicht-Erwachsenen? Strafrecht und Jugendschutzrecht unterscheiden 
- Kinder (0-13 Jahre),

- Jugendliche (14-17 Jahre) und

- Heranwachsende (18-20 Jahre) [3, 4].

Das Bürgerliche Gesetzbuch hingegen verwendet den Begriff

- Minderjähriger.

Dieser umfasst alle Personen, die das 18. Lebensjahr noch nicht vollendet haben. Ronny ist daher sowohl jugendlich als auch minderjährig - je nach Rechtsgebiet.

Einwilligungsfähigkeit Damit der Eingriff in die körperliche Integrität eines Patienten gerechtfertigt ist, muss dieser grundsätzlich in die Behandlung einwilligen. Denn ohne eine wirksame Einwilligung gilt auch ein indizierter ärztlicher Eingriff generell als Körperverletzung und ist somit strafbar - selbst bei fehlerfreier Behandlung [5-10].

Ronny war zunächst mit der ärztlichen Behandlung einverstanden - er nahm ein Schmerzmittel ein und war auch zu einer Behandlung mit Antiserum bereit. Aber:

- Kann ein Minderjähriger eigenständig rechtsgültig in die Behandlung einwilligen?

- Kann er den Schweregrad seiner Erkrankung sowie die empfohlene Therapie mit ihren Chancen und Risiken selbst bewerten?

Es gibt diesbezüglich keine eindeutige Regelung.

Für die Einwilligungsfähigkeit Minderjähriger sind keine gesetzlich definierten Altersgrenzen vorgegeben.

Die Internistin musste also Ronnys Einwilligungsfähigkeit selbst einschätzen. In diesem Fall schien ihr der Patient verständig genug, um das Geschehen aus Schlangenbiss, möglichen Folgen und therapeutischen Optionen einordnen zu können.
Beurteilung durch den Arzt Maßgebend für die Einwilligungsfähigkeit sind Einsicht und Urteilsvermögen des Minderjährigen: Durch Einsichtsfähigkeit ist es möglich, Zusammenhänge zu verstehen und in sinnvoll oder sinnlos, nötig oder unnötig einteilen zu können. Je komplexer die Zusammenhänge, desto größer das erforderliche Abstraktionsvermögen. Daher braucht es Jahre an Entwicklung, bis eine Person tatsächlich eigenständig verstehen und urteilen kann. Leider ist diese Fähigkeit nicht messbar [11, 12].

- Als Richtgröße kann das 15. Lebensjahr gelten. Das 14. Lebensjahr sollte nicht unterschritten werden.

- Die sehr individuelle persönliche Entwicklung lässt eine exakte Altersangabe jedoch nicht zu [13].

Nach der Rechtsprechung des Bundesgerichtshofs kommt es allgemein darauf an, ob der Jugendliche die Bedeutung und Tragweite des Eingriffs ermessen kann $[14,15]$.

Die Einwilligungsfähigkeit des jungen Patienten ist also stets im Einzelfall zu ermitteln.

Aufklärung Mit der Einwilligungsfähigkeit ist auch die Frage verbunden, an wen der Arzt das Aufklärungsgespräch richten muss.

- Liegt keine Einwilligungsfähigkeit vor, so sind auf jeden Fall die gesetzlichen Vertreter die Ansprechpartner ( $\bullet$ Kasten S. 12).

- Gleiches gilt bei nur zweifelhafter Einwilligungsfähigkeit.

- Ist der Minderjährige einwilligungsfähig, ist die Rechtslage umstritten.

Gemäß der Rechtsprechung des Bundesgerichtshofs genügt im Allgemeinen die Aufklärung und Einwilligung der Eltern.

\section{Zustimmung der gesetzlichen Vertreter}

Gesetzliche Vertreter sind in der Regel beide Eltern [23, 25]. Für den Fall, dass nur ein Elternteil anwesend ist, hat der Bundesgerichtshof eine „Dreistufentheorie“ entwickelt [24]. Danach hängt das korrekte Vorgehen des Arztes von der Tragweite der Entscheidung ab:

1. Allgemeiner Routinefall: Fehlen gegenteilige Informationen, kann der Arzt von der Einwilligung des nicht erschienenen Elternteils ausgehen.

2. Eingriffe schwererer Art mit nicht unbedeutenden Risiken: Der Arzt muss der Frage nachgehen, ob und wieweit der fehlende Elternteil den erschienenen zur Zustimmung ermächtigt hat. Er kann sich aber trotzdem auf die positive Antwort des erschienenen Elternteils verlassen.

3. Schwierige und weitreichende Entscheidungen mit erheblichen Risiken für das Kind: Hier hat sich der Arzt Gewissheit über die Zustimmung beider Eltern zu verschaffen.

Besteht für das Wohl des nicht einwilligungsfähigen Kindes „Gefahr im Verzug“, kann die Einwilligung notfalls von nur einem Elternteil erklärt werden [25].

Allerdings steht dem Minderjährigen in Einzelfällen ein Vetorecht zu - zum Beispiel, wenn der Eingriff erhebliche Folgen für seine künftige Lebensgestaltung haben kann [16]. Dieses Veto kann einen aufschiebbaren medizinischen Eingriff verzögern, bis der Minderjährige die nötige Entscheidungskompetenz erlangt [17].

Damit der Minderjährige von diesem Vetorecht Gebrauch machen kann, sollte der Arzt auch ihn aufklären [16].

Je dringlicher und unaufschiebbarer die Behandlung ist, desto weniger Zeit darf durch die Information der Eltern versäumt werden.

- In Eil- und Notfällen reicht in aller Regel die Einwilligung des Minderjährigen.

- Auch kann der Arzt die indizierte Behandlung unter dem Gesichtspunkt der mutmaßlichen Einwilligung oder des Notstands durchführen $[18,19]$.

Ablehnung der Behandlung Dr. Windfuhr hielt Ronny für einwilligungsfähig. Die Entscheidung für oder gegen die Therapie lag daher auch bei ihm. Dieses Recht hat Ronny dann auch umgesetzt, als er sich, nach ordnungsgemäßer Aufklärung, gegen die Antidot-Behandlung und gegen einen weiteren Krankenhausaufenthalt aussprach. Die Ärztin versuchte richtigerweise, ihren minderjährigen Patienten umzustimmen - was ihr jedoch nicht gelang. Im vorliegenden Fall war die Dringlichkeit zur Gabe des Antidots noch nicht derart groß, dass unmittelbar Handlungsbedarf bestand.

Die Entscheidung, Ronny gegen dringenden ärztlichen Rat und mit konkreten Verhaltensanweisungen nach Hause gehen zu lassen, erscheint daher riskant, aber vertretbar.

Anders kann das bei dringendem Handlungsbedarf aussehen ( $\bullet$ Kasten S. 13).

Schweigepflicht Grundsätzlich gilt die ärztliche Schweigepflicht auch bei Minderjährigen [13]. Das umfasst bereits die Tatsache, dass jemand in Behandlung ist.

- Gegen den Willen eines voll einsichtsund urteilsfähigen Minderjährigen darf der Arzt die Eltern im Regelfall also nicht informieren.

- Allerdings kann ein einwilligungsfähiger Minderjähriger den Arzt von seiner Schweigepflicht entbinden. 
Nur das unbefugte Offenbaren von Patientengeheimnissen ist strafbar $[20,21]$.

Eine kurze Frage hätte also die Unsicherheit beseitigen können: „Ich würde jetzt mal deine Eltern anrufen - ist das in Ordnung für dich?" Vielleicht wäre Ronny mit der Kontaktaufnahme zu seiner Mutter ohnehin einverstanden gewesen.

- Die Entbindung von der Schweigepflicht muss der Arzt vorab einholen; eine nachträgliche Zustimmung gibt es im Strafrecht nicht.

Ronny hatte nicht widersprochen, als die Ärztin seine Mutter in das Aufklärungsgespräch einbezog. Insofern hat er die Ärztin zumindest konkludent von der Schweigepflicht entbunden.

Dokumentation Barbara Windfuhr entließ ihren Patienten gegen ausdrücklichen ärztlichen Rat - und notierte die Non-Compliance sowie die eingehende Beratung ausführlich in der Patientenakte. Ein wichtiger Schritt, denn neben der

- berufsrechtlichen Pflicht zur Dokumentation [22]

- schützt eine exakte Aufzeichnung vor späteren rechtlichen Unklarheiten.

Es gilt allerdings:

Der Patient kann nicht gezwungen werden, sein Handeln gegen ärztlichen Rat mit seiner Unterschrift zu bestätigen. Ein Formular wie im Fallbeispiel ist daher nicht obligat.

Eine detaillierte Dokumentation des gesamten Behandlungsablaufs schafft für alle Beteiligten die Sicherheit, die einzelnen Schritte auch im Nachhinein verfolgen zu können. Notieren Sie daher nicht nur,

- dass Sie bestimmte Entscheidungen getroffen haben, sondern auch,

- warum Sie sich für diesen Weg entschieden haben.

Schreiben Sie dabei leserlich!

Wiedersehen Ronny erscheint am nächsten Tag erneut im Krankenhaus. Die Schmerzen haben wieder zugenommen, kombiniert mit Schwindelgefühl und Übelkeit. Er akzeptiert die Gabe des Antiserums. Nach vorsorglicher weiterer Überwachung über wenige Stunden können die Ärzte ihn ohne weitere Nachwirkungen des Giftes oder des Antiserums nach Hause entlassen.
Fazit Auch wenn Sie im Notfall schnell entscheiden müssen: Bei der medizinischen Behandlung Minderjähriger sind stets rechtliche Aspekte zu berücksichtigen. Handeln Sie diesbezüglich nicht sorglos - eine ärztliche Behandlung kann sonst leicht

- zivilrechtliche,

- berufsrechtliche oder sogar

- strafrechtliche Folgen haben.

Bettina Rakowitz, Jörg Bossenmayer

\section{Literatur online}

Das vollständige Literaturverzeichnis zu diesem Beitrag finden Sie im Internet:

Abonnenten und Nichtabonnenten können unter „www.thieme-connect.de/ejournals" die Seite der Lege artis aufrufen und beim jeweiligen Artikel auf „Ergänzendes Material“ klicken - hier ist die Literatur für alle frei zugänglich.

Abonnenten können alternativ über ihren persönlichen Zugang an das Literaturverzeichnis gelangen. Wie das funktioniert, lesen Sie unter: http://www.thiemeconnect.de/ejournals/help\#SoRegistrieren

Beitrag online zu finden unter http://dx. doi.org/10.1055/s-0031-1272348

\section{Kernaussagen}

- Patienten, die das 18. Lebensjahr noch nicht vollendet haben, sind minderjährig. Das heißt jedoch nicht, dass in jedem Fall nur der gesetzliche Vertreter für den Patienten sprechen darf - dem Minderjährigen steht eventuell zumindest ein Vetorecht zu.

- Ein Minderjähriger kann unter Umständen durchaus eigenständig in eine ärztliche Behandlung einwilligen. Ob das der Fall ist, müssen Sie als Arzt beurteilen: Wenn Sie Ihrem minderjährigen Patienten zutrauen, die Situation umfassend zu verstehen, dann können Sie ihm auch die Fähigkeit zur Einwilligung zutrauen.

- Ob einwilligungsfähig oder nicht: Binden Sie den Minderjährigen in das Aufklärungsgespräch ein und berücksichtigen Sie dessen Argumente.

- Die ärztliche Schweigepflicht gilt grundsätzlich auch gegenüber den Eltern des Minderjährigen! Fragen Sie deshalb nach: Ist der Minderjährige mit der Benachrichtigung der Eltern einverstanden? Dann sind Sorgen wegen einer möglichen Verletzung der Schweigepflicht unbegründet.

- Dokumentieren Sie den gesamten Ablauf inklusive aller Entscheidungen - einschließlich der zugrunde liegenden Überlegungen.

\section{Was tun bei unmittelbarem Handlungsbedarf?}

Grundsätzlich darf der Arzt den Willen eines einwilligungsfähigen Jugendlichen nicht übergehen - eine ordnungsgemäße Aufklärung vorausgesetzt. Andererseits ist der Arzt als sogenannter Garant grundsätzlich zur Hilfe verpflichtet. Bei unmittelbarem Handlungsbedarf sollte der Arzt daher folgendermaßen vorgehen:

- Lehnt der einwilligungsfähige Jugendliche die dringend notwendige Behandlung ab, die anwesenden Eltern(teile) stimmen der Behandlung aber zu, muss der Arzt versuchen, den Jugendlichen umzustimmen. Gelingt dies nicht, geht nach herrschender Ansicht das elterliche Sorgerecht vor $[14,16]$. Die Behandlung sollte vorgenommen werden - notfalls mit Hilfe des Familiengerichts.

- Lehnen sowohl die Eltern als auch der Jugendliche oder nur die Eltern die dringend notwendige Behandlung ab, muss der Arzt versuchen, die Eltern und ggf. den Jugendlichen umzustimmen. Gelingt dies nicht, muss der Arzt das Familiengericht einschalten.

Das Familiengericht ist eine Abteilung des Amtsgerichts. In jedem Amtsgericht ist ein Richter im Bereitschaftsdienst erreichbar - auch nachts und am Wochenende. Schildern Sie diesem den Fall; er entscheidet über das weitere Vorgehen.

- Erkundigen Sie sich, welches Amtsgericht für Ihr Krankenhaus zuständig ist. Notieren Sie sich Namen und Telefonnummern und verwahren Sie sie griffbereit für den Notfall. 